\title{
Preparation and Characterization of Epoxidised and Acrylated Styrene/Isoprene/Styrene (SIS) Triblock Block Copolymer Based Nanocomposites
}

\author{
Santosh Khanal, Alina Shakya ${ }^{1}$, Goerg H. Michler ${ }^{2}$, Boulos Youssef ${ }^{3,4}$, \\ Jean M. Saiter ${ }^{3}$, Rameshwar Adhikari ${ }^{1, *}$ \\ ${ }^{1}$ Central Department of Chemistry, Tribhuvan University, Kathmandu, Nepal. ${ }^{2}$ Institute of Physics, \\ Martin Luther University Halle-Wittenberg, D - 06099 Halle/Saale, Germany. ${ }^{3}$ AMME-LECAP \\ International Lab EA 4528 Université de Rouen, 76801 Saint Etienne du Rouvraycedex, France. ${ }^{4}$ INSA \\ de Rouen, 76801 Saint Etienne du Rouvraycedex, France, \\ E mail: nepalpolymer@yahoo.com
}

\begin{abstract}
In this work, a commercially available Styrene-Isoprene-Styrene (SIS)triblock copolymer was modified into epoxidised version (ESIS)using performic acid generated in situ from hydrogen peroxide and formic acid. The epoxidised sample was further acrylated to prepare acrylated version (ASIS). The nanocomposites of each sample (SIS, ESIS and ASIS) were prepared using boehmite nanoparticles as filler by solution casting method. The polymers were characterized by Fourier Transform Infrared (FTIR) spectroscopy and transmission electron microscopy (TEM). TEM investigations revealed that that the epoxidation of the diene block enhanced the dispersion of the nanofiller in the polymer matrix while the segregation of the nanoparticles towards the interface of the immiscible polymers was observed in the acrylated block copolymer based nanocomposite.
\end{abstract}

Keywords: SIS triblock copolymer, epoxidation, acrylation, FTIR spectroscopy, TEM

\section{Introduction}

Styrene-Isoprene-Styrene (SIS) triblock copolymers belong to the class of thermoplastic elastomers whose structures are characterized by microphase-separation of the constituent chains ${ }^{1,2}$. The SIS copolymers behave as cross-linked rubbers at ambient temperatures while are processable as thermoplastics at elevated temperatures ${ }^{2}$. They have several useful properties such as high strength, excellent elasticity and low temperature durability. However, these are non-polar polymers and have low resistance to hydrocarbon oils or several solvents. Different functional groups can be introduced to this polymer to increase molecular polarity. On the other hand, anionic polymerization technique used to synthesize SIS block polymer is not much organized to incorporate polar monomers during polymerization. Due to this constraint, chemical modification to generate polar group on block polymer appears to be a viable and effective approach ${ }^{3}$. Among various chemical modifications, epoxidation is a convenient one to incorporate oxygen in diene block. By controlling the degree of epoxidation, properties of these materials can be altered. More ever, epoxide ring being reactive and unstable, ring opening reaction can be carried out in it ${ }^{4}$. Some methods were described for preparing epoxidised polymer using dioxirane $^{5}, \mathrm{~m}$-chloroperoxy ${ }^{6}$ and in situ generated peracetic acid ${ }^{7}$. Among various oxidants used to perform the epoxidation reactions, the in situ generated organic peracid has been proved to be a better option as it overcomes disadvantages of the peracids such as their instability, inconvenience in storage,

\footnotetext{
*Corresponding author
} 
and their ease to decompose. Although the polar modified versions of the styrene/diene block copolymers by selective epoxidation of the diene block are employed for preparing compounds with other polymers or fillers, it is also of interest to investigate the effect of such modifications on their physico-mechanical properties. Recently, such studies have been extended to styrene/butadiene block copolymers having asymmetric architectures and possessing new morphologies at equilibrium ${ }^{7}$.

In this study, SIS triblock copolymer is epoxidised using performic acid generated in situ by the reaction of formic acid and hydrogen peroxide at $60^{\circ} \mathrm{C}$.Small amount of polyethylene glycol (PEG) as phase transfer catalyst is also added to facilitate mixing of formic acid and hydrogen peroxide which are otherwise immiscible. The epoxidised sample was subjected to ring opening reaction using acrylic acid. These samples were used to prepare nanocomposites with boehmite nanoparticles which were then characterized by Fourier transform infrared (FTIR) spectroscopy and transmission electron microscopic (TEM) analysis.

\section{Experimental Methods}

\section{Materials}

Styrene-isoprene-styrene (SIS) triblock copolymer was a commercial product of Kraton Polymers under the trade name Kraton D1160CS containing $19 \mathrm{wt}-\%$ of polystyrene (PS). The number average molecular weight and polydispersity index of the polymer used as determined by gel permeation chromatography using PS as standard were found to be $178,000 \mathrm{~g} / \mathrm{mol}$ and 1.30 respectively. The triblock copolymer sampler was kindly provided by Nordmann-Rasmann GmbH, Hamburg, Germany. The $p$ toluene sulfonic acid coated boehmite nanoparticles were kindly provided by Sasol Chemical Company, Hamburg. Hydrogen peroxide (30 wt.-\%) formic acid (85\% wt.-\%), acrylic acid, and other chemicals were used as received from the local market in Kathmandu.

\section{Preparation of Epoxidised (ESIS) Block Copolymer}

Epoxidation of SIS was carried by dissolving $5 \mathrm{~g}$ of polymer in $100 \mathrm{ml}$. of toluene keeping the solution still for $24 \mathrm{hrs}$ before followed by heating to $60{ }^{\circ} \mathrm{C}$. Then $1.368 \mathrm{~g}$ of formic acid and $1.609 \mathrm{~g}$ of hydrogen peroxide were added sequentially along with $1 \%$ by weight of total polymer of polyethylene glycol (PEG) as phase transfer catalyst. The mixture was left for $2 \mathrm{hrs}$ and was precipitated with methanol, washed and dried. The reaction is presented in scheme 1.

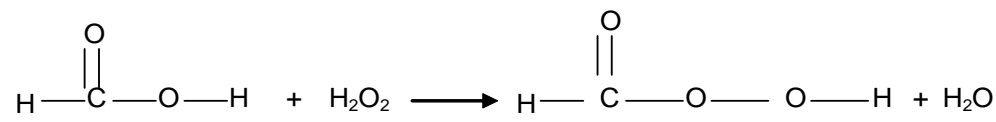
Formic acid
Hydrogen peroxide
Performic acid

Performic acid generated by reaction of formic acid and hydrogen peroxide

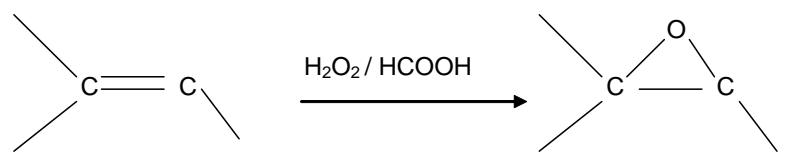

Isoprene Unit Epoxide Unit

Epoxidation of isoprene unit

Scheme 1: Epoxidation of isoprene units of the SIS triblock copolymer 


\section{Preparation of Acrylated (ASIS) Block Copolymer}

$2.5 \mathrm{~g}$ of epoxidised ESIS was dissolved in $50 \mathrm{ml}$. of toluene taking in a round bottom flask. $5 \mathrm{~g}$ of acrylic acid was added drop-wise and mixture as stirred and kept at $110^{\circ} \mathrm{C}$ for $3 \mathrm{hrs}$ and was precipitated by methanol, then washed and dried. Thus, the ESIS was subjected to ring opening reaction with acrylic acid. The reaction is depicted in Scheme 2.

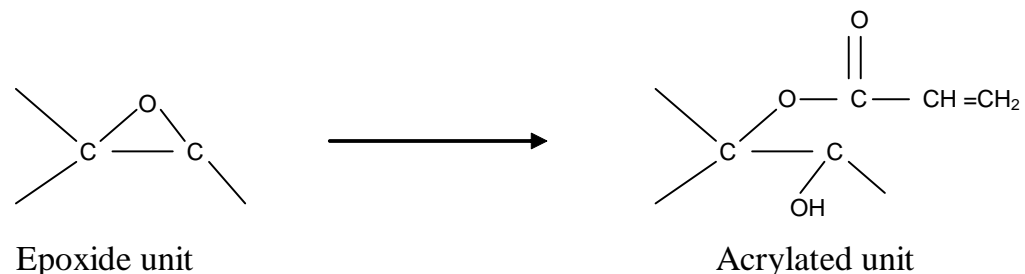

Scheme 2: Acrylation of epoxide units present in ESIS

\section{Preparation of Polymer Nanocomposites}

The nanocomposites of SIS, ESIS and ASIS with boehmite nanofiller ( $95 / 5$ by weight) were prepared by solution casting method using cyclohexane as solvent.

\section{Characterization Techniques}

Fourier Transform Infrared (FTIR) spectroscopy and transmission electron microscopy (TEM) were used for the characterization of the structure and morphology of the sample. FTIR spectra were collected by a Perkin Elmer FTIR spectrophotometer in ATR mode by collecting and averaging 32 scans over a range of $400 \mathrm{~cm}^{-1}-4000 \mathrm{~cm}^{-1}$ at a resolution of $4 \mathrm{~cm}^{-1}$. TEM measurements were performed on ultrathin sections of the samples (approximately $60 \mathrm{~nm}$ thick) sectioned from the bulk specimen by means of a Leica Cryo-ultramicrotome equipped with a diamond knife.

\section{Results and Discussion}

\section{Preliminary Characterization}

The amount of epoxy group in epoxidised SIS block copolymer (ESIS) was determined titrimetrically by $\mathrm{HCl} /$ acetone method ${ }^{9}$. In a typical experiment, $1 \mathrm{~g}$ of sample was treated with $25 \mathrm{ml}$ of $\mathrm{HCl} /$ acetone (1:80 by volume) mixture and stirred until the sample formed a homogeneous solution. The resulting solution was titrated with standardized alcoholic caustic soda $(\mathrm{NaOH})$ solution using phenolphthalein as indicator. The epoxy value is calculated as ${ }^{9}$ :

$$
\text { Epoxy value }(E)=\frac{\left(\mathrm{V}_{0}-\mathrm{V}_{1}\right) \cdot \mathrm{N}}{10 \mathrm{~W}}
$$

where $\mathrm{V}_{1}, \mathrm{~V}_{0}, \mathrm{~N}$ and $\mathrm{W}$ stand for volume of $\mathrm{NaOH}$ used for given sample (1), reference sample (0), normality of the $\mathrm{NaOH}$ solution and weight of sample in gram, respectively. The titrimetric analysis showed that the ESIS sample contained $0.294 \%$ mole of epoxy group which is equivalent to $25 \%$ of total isoprene unit. The observation is also consistent with earlier reports by Li et al. ${ }^{10}$.

\section{Fourier Transform Infrared (FTIR) Spectroscopic Characterization}

The FTIR spectra of ESIS and ASIS are compared with that of neat SIS triblock copolymer in Fig. 1. At the first glance, one can notice several changes in the spectra of epoxidised and acrylated samples 
relative to that of the neat SIS. The spectrum of the ESIS shows the characteristic peaks of oxirane ring at centered at $870 \mathrm{~cm}^{-1}$ for (corresponding to half epoxy ring stretching) and at $1270 \mathrm{~cm}^{-1}$ (corresponding to the whole epoxy ring stretching $)^{3,4}$. This confirms the formation of epoxidised SIS. In addition, there appears a peak centered at $1731 \mathrm{~cm}^{-1}$ that corresponds to the $>\mathrm{C}=\mathrm{O}$ group stretching implying that some side reaction occurred during the experiment.

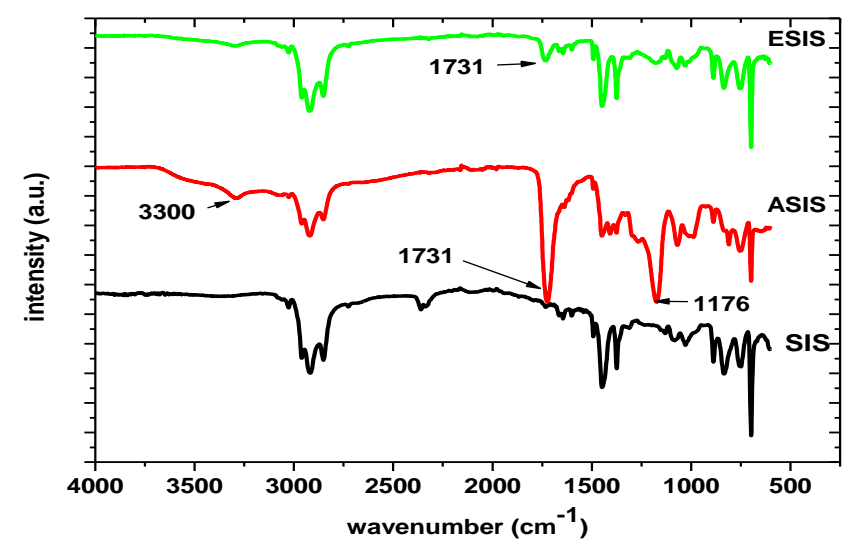

Figure 1: FTIR spectra of SIS, ESIS and ASIS Copolymers

Further the spectrum of acrylated SIS copolymer (ASIS) shows the peaks centered at $3300 \mathrm{~cm}^{-1}$, $1731 \mathrm{~cm}^{-1}$ and $1176 \mathrm{~cm}^{-1}$ which indicates clearly the presence of $-\mathrm{OH},-\mathrm{C}=\mathrm{O}$ groups and ester linkage, respectively in the ASIS. Thus, this observation confirms the grafting of acrylic acid in ESIS.

\section{Transmission electron microscopic (TEM) Analysis}

One of the prime objectives of this work was to analyze the effect of various chemical modification of the SIS triblock copolymer on the structure and properties of nanocomposites. For this purpose, the samples were blended with $p$-toluene sulphonic acid modified boehmite nanoparticles with the polymers. We present here the morphology of the nanocomposites comprising SIS and ESIS as matrix. Fig. 2 shows the TEM micrographs of the nanocomposite of SIS/5 wt.-\% boehmite nanocomposite.
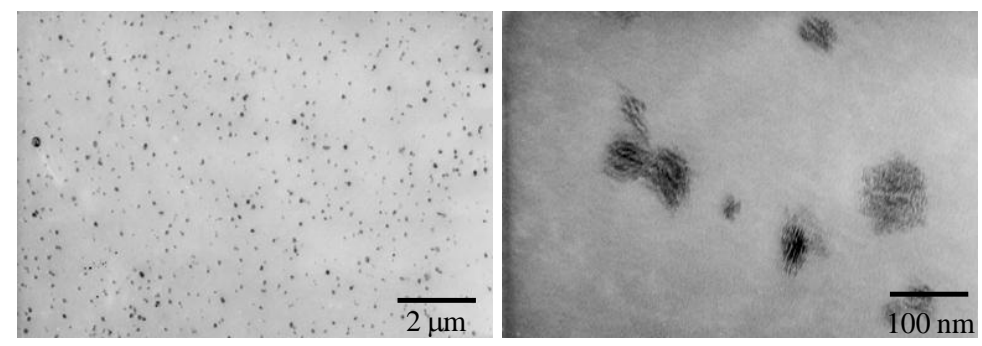

Figure 2. Lower (left) and higher (right) TEM images of SIS/5 wt.-\% boehmite nanocomposite

In TEM image the inorganic particles appear as dark spots while the organic polymer matrix appears as brighter areas. It can be easily identified that the nanometric filler has the sphere shaped particle with diameter ranging from few tens of nanometers to about $100 \mathrm{~nm}$. The particles have distributed quite uniformly throughout the polymer matrix and the distribution of particle size is also narrow. 
TEM micrographs showing the morphology of ESIS/5 wt.-\% filler are presented in Fig. 3. The first comparative observation between Figs. 2 and 3 makes it evident that the density of dark appearing particles is higher in Fig. 3 than in Fig. 2 while the size of the particles and their dispersion into individual crystals appears more pronounced in the nanocomposite of boehmite with epoxidised block copolymer sample. This can be explained by the affinity of the polar group containing filler nanoparticles with more polar version of the block copolymer (i.e. ESIS) compared to neat SIS triblock copolymer.

Nevertheless, the effect is not dramatic. It may be expected that more uniform and finer dispersion of the nanoparticles may be obtained in the block copolymer which is more strongly polar modified than in the present study.
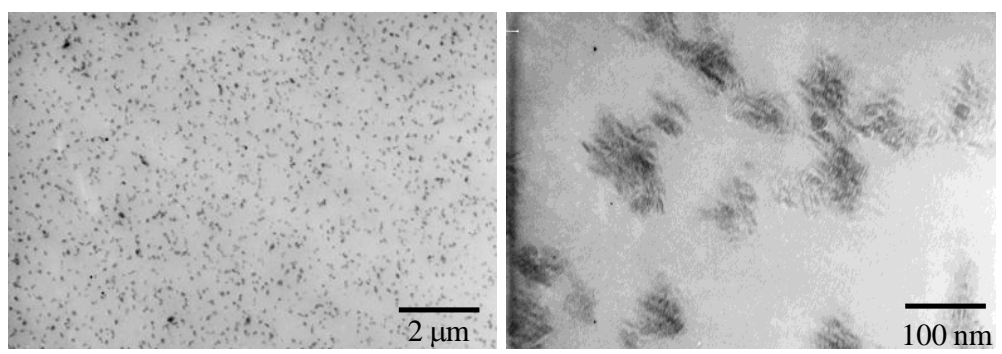

Figure 3. Lower (left) and higher (right) TEM images of ESIS/5 wt.-\% boehmite nanocomposite

\section{Conclusions}

The styrene-isoprene-styrene (SIS) tri block copolymer was successfully epoxidised by performic acid formed in situ by the action of formic acid and hydrogen peroxide. The epoxidised polymer is further acrylated and nanocomposites of the polymer with boehmite nanofiller. A slight improvement in the dispersibility of the nanofiller was observed due to polar modification of the SIS block copolymer. The influence of polar modification of the block copolymer and subsequent fabrication of nanocomposites with different kinds of fillers on physico-mechanical properties of the compounds shall be the object of future studies.

\section{Acknowledgements}

The authors thank Nepal Academy of Science and Technology (NAST) for providing a research promotion grant. R. A. acknowledges Alexander von Humboldt (AvH) Foundation for providing support during his research visits to Germany on different occasions.

\section{References}

1. I. Hamley, The Physics of Block Copolymers, Oxford Publishers, London, 1998.

2. G. Hoden, Understanding Thermoplastic Elastomers, Carl Hanser Verlag, Munich, 2011.

3. K. Udipi, J. Appl. Polym. Sci., 1979, 23, 3301.

4. A. Chaisuriyathepkul, P. Klinpituksa, P. Phinyocheep, C. Nakason and S. Kittipoon, e-Polymers 2008, No. 141.

5. R. B. Grubbs, M. E. Broz, J. M. Dean and F. S. Bates Macromolecules, 2000, 33, 2308.

6. M. Antonietti, S. Forster, J. Hartman, S. Oesteich, Macromolecules, 1996, 29, 3800.

7. S. M. Wang and R. C. Tsiang, J. Polym. Sci. Part A: Polym. Chem., 1996, 34, 1483.

8. R. Pandit, J. Giri, R. Lach, W. Grellmann, G. H. Michler, B. Youssef, J. M. Saiter and R. Adhikari, Macromol. Symp., 2012, 315, 152.

9. S. Siggia and J. G. Hanna, Quantitative Organic Analysis via Functional Group, $4^{\text {th }}$ Edition, John Wiley \& Sons, New York, 1979.

10. H. Li, X. Zeng and W. Wu, J. Elastom. Plast., 2008, 40, 317. 